Einführung in das Schwerpunktthema

\title{
Kurs Nachhaltigkeit? Perspektiven der Förderung von Nachhaltigkeitsforschung
}

S Von Thomas Korbun eit dem Weltgipfel für Umwelt und Entwicklung in Rio im Jahre 1992 hat das Leitbild einer Nachhaltigen Entwicklung zunehmend Eingang in die politische Entscheidungsfindung und in die Wissenschaft gefunden. Die Nationale Nachhaltigkeitsstrategie der Bundesregierung ist ein Ausdruck dieser Entwicklung. In der Wissenschaft führte die zunehmende Bedeutung der Nachhaltigkeit zur Herausbildung eines neuen Forschungstyps, der transdisziplinären Nachhaltigkeitsforschung. Er versteht sich als neue Form der ,Wissensproduktion', der Natur- und Sozialwissenschaften übergreifend, problemorientiert, akteursorientiert und transdisziplinär arbeitet. Neben wissenschaftlichem Wissen stellt Nachhaltigkeitsforschung auch Wissen für gesellschaftliche Entscheidungsprozesse bereit und dient als Ressource für die Lösung komplexer sozial-ökologischer Problemlagen.

Mit dem Rahmenprogramm Forschung für die Nachhaltigkeit (FONA) hat das Bundesministerium für Bildung und Forschung (BMBF) einen paradigmatischen Wandel von der Förderung einer disziplinär verengten Umweltforschung zu einer transdisziplinären Nachhaltigkeitsforschung vollzogen (1). Der Schwerpunkt dieses Heftes beleuchtet den neuen Forschungstyp und diskutiert Perspektiven, die mit dem neuen Rahmenprogramm verbunden sein können. Eine besondere Rolle nimmt die internationale Dimension ein, durch den Vergleich mit anderen nationalen Förderprogrammen zur Forschung für eine Nachhaltige Entwicklung und durch einen kritischen Blick auf die Forschungsförderung der Europäischen Union.

\section{- Die Beiträge im Überblick}

Thomas Jahn zeichnet in seinem Beitrag die Hintergründe der Entstehung der transdisziplinären Nachhaltigkeitsforschung in Deutschland nach und beschreibt ihre Charakteristika. Anhand des BMBF-Förderschwerpunktes ,,Sozialökologische Forschung“ zeigt der Beitrag welche
Erfahrungen mit diesem Forschungstyp im Rahmen der BMBF-Förderung bislang vorliegen und zieht daraus Schlussfolgerungen für die Umsetzung von FONA.

Eine besondere Verantwortung der Wissenschaft für die Umsetzung einer Nachhaltigen Entwicklung betont der Nachhaltigkeitsrat der Bundesregierung. In den letzten Monaten haben Vertreter des Rates wiederholt beklagt, die Wissenschaft lege „noch nicht in ausreichendem Maße Beiträge zur Nachhaltigkeit" vor (2). Der Rat begrüßt daher die Verabschiedung von FONA und verspricht sich dadurch Impulse in Wissenschaft und Gesellschaft. Angelika Zahrnt benennt in ihrem Beitrag als Mitglied des Rates gesellschaftliche Anforderungen an die Nachhaltigkeitsforschung, mahnt einen Wandel der forschenden Institutionen zu mehr Interdisziplinarität an und fordert die Forschenden auf, sich aktiver an den gesellschaftlichen Dialogen zu beteiligen.

Auch in anderen Ländern hat die staatliche Forschungsförderung den Paradigmenwechsel zu Nachhaltigkeitsforschung eingeleitet. Kathy Whitelegg gibt in ihrem Beitrag einen Überblick über die Forschungsprogramme für Nachhaltigkeit in ausgewählten Mitgliedsstaaten der Europäischen Union. In den unterschiedlichen Erfahrungen liegt ein erhebliches Lernpotenzial für die Gestaltung von Forschungsprogrammen. Grundsätzlich lassen sich zwei Typen unterscheiden: einerseits ein eher loses Nebeneinander verschiedener thematischer Programme mit Nachhaltigkeitsbezug, andererseits Rahmenprogramme, die - ähnlich wie FONA - neben Forschungen zu verschiedenen thematischen Feldern eine Weiterentwicklung des Forschungsansatzes und eine Stärkung der Forschungskapazitäten auf diesem vergleichsweise jungen Forschungsgebiet anstreben. Antonia Wenisch und Céline Loibl zeigen am Beispiel Österreichs genauer, dass der Weg zu einer übergreifenden Forschungsstrategie auch von Vorbehalten und Widerständen begleitet sein kann. Der industriedominierte Forschungs- und Technologierat hat nach langen Diskussionen im Sommer 2004 doch noch eine übergreifende Strategie für die österreichische Nachhaltigkeitsforschung beschlossen.

Mit dem Leitbild der Nachhaltigen Entwicklung eng verbunden ist eine globale Perspektive auf sozial-ökologische Problemlagen und Vorschläge zu ihrer Lösung. Folgerichtig wird ein solcher Themenzugang von Forschungseinrichtungen und Projekten der Nachhaltigkeitsforschung zunehmend erwartet, ebenso das Arbeiten in vielfältigen internationalen Kooperationen. Ein Blick auf die Forschungsförderung der Europäischen Union macht aber deutlich, dass die fortschrittlichen nationalen Initiativen dort nicht die dafür unabdingbare Fortsetzung finden. Nina Sartori legt mit ihrem Beitrag eine Bestandsaufnahme der wenigen Fördermöglichkeiten für ansatzweise transdisziplinäre, Natur- und Sozialwissenschaften übergreifende Forschung zu Nachhaltiger Entwicklung in dem aktuell laufenden sechsten EU-Forschungsrahmenprogramm vor (3). Ihr Ausblick auf das kommende siebte Rahmenprogramm stimmt in dieser Hinsicht noch pessimistischer: Nach ersten Verlautbarungen der Kommission wird sich der schon bislang sichtbare Trend der Fokussierung auf disziplinär enger geführte Technologieentwicklung noch deutlich verstärken.

Ein weiteres Hindernis für die Nachhaltigkeitsforschung stellt die Begrenzung der Förderung auf wenige sehr große Forschungscluster dar. Innovative Forschung zu Nachhaltiger Entwicklung wird in einigen Mitgliedsstaaten maßgeblich von kleineren Forschungseinrichtungen geprägt, die oft ohne staatliche Grundförderung auskommen müssen. Deren Beteiligungschancen an der europäischen Forschungszusammenarbeit sinken dadurch weiter. Neben der Einrichtung von FONA auf nationaler Ebene muss die Bundesregierung daher auch bei Initiativen für eine bessere Verankerung der Nachhaltigkeitsforschung in Europa eine erkennbare Vorreiterrolle einnehmen. Die Stellungnahme der Bundesregierung zum siebten Rahmenprogramm wird dieser Erwartung jedoch nicht gerecht.

Parallel sollte sie sich verstärkt bei der Zusammenarbeit und Koordination der nationalen Förderprogramme im Europäischen Forschungsraum engagieren und multilaterale Förderbekanntmachungen in ausgewählten Themenfeldern entwickeln. Die fortschrittlicheren Mitgliedsstaaten könnten so Maßstäbe für eine zukünftige gesamteuropäische Forschungsförderung zu Nachhaltiger Entwicklung setzen. 


\section{- Nagelprobe Förderpraxis}

Ein Konzept wie FONA ist eine Voraussetzung für die Entwicklung und Stärkung einer erfolgreichen Nachhaltigkeitsforschung. Weitere förderpraktische Aspekte kommen hinzu. Die Förderbekanntmachungen zu einzelnen Themen im Rahmen von FONA müssen den Übergang von engerer Umwelt- zu Nachhaltigkeitsforschung mit vollziehen. Mehrere Bekanntmachungen dieses Jahres haben sich bereits vor der Verabschiedung von FONA auf das erwartete Rahmenprogramm bezogen. Der Förderschwerpunkt ,Nachhaltige Waldwirtschaft" markiert beispielsweise eine ambitionierte Weiterentwicklung der bisherigen Förderung zu Wald- und Holzwirtschaft in Richtung einer transdisziplinären Nachhaltigkeitsforschung. Weniger deutlich bleibt der Wechsel im Förderschwerpunkt „Innovationen als Schlüssel für Nachhaltigkeit in der Wirtschaft", der vordergründig auf technische Innovationen setzt, allerdings auch eine gewisse Öffnung hin zu sozialen und institutionellen Innovationen erkennen lässt.

Welche der eingereichten Projekte zur Förderung kommen, bestimmen maßgeblich die berufenen Gutachter. Ihrer Auswahl und Vorbereitung kommt eine Schlüsselrolle bei der Umsetzung von FONA zu. Dominieren in den Gremien die klassischen Forschungsakteure, ist die Gefahr groß, dass ambitionierte Programme dennoch nur klassische, stärker an einzelnen Disziplinen orientierte Forschungsvorhaben hervorbringen. Dies war im fünften EU-Rahmenprogramm gut zu beobachten: konzeptionell nicht mehr auf Technologiebereiche, sondern auf die integrierte Lösung gesellschaftlicher Probleme ausgerichtet, dominierten trotzdem disziplinär verengte, techniklastige Projekte. Weiter gefasste, interdisziplinäre Projekte scheiterten in hohem Maße an den klassisch besetzten Gutachtergremien.

Nach der Einrichtung von Förderschwerpunkten kam es in der Vergangenheit zu einer Reihe von projektiibergreifenden Querschnittsaktivitäten. Sie hatten das Ziel, übergreifende thematische und methodische Fragen zu bearbeiten, die Projektergebnisse synoptisch zusammenzufuihren und zukünftige Forschungsbedarfe zu identifizieren. Beispiele sind die Förderschwerpunkte ,Betriebliche Instrumente für nachhaltiges Wirtschaften" oder - mit einem konzeptionell innovativen, besonders breiten Maßnahmenspektrum - ,Sozial-ökologische Forschung“ (4). Vera Rabelt fasst in ihrem Beitrag beispielhaft die Erfahrungen in dem abgeschlossenen Förderschwerpunkt „Neue Nutzungsstrategien - Regionale Ansätze“ zusammen. Dabei wird

\section{FONA in Kürze}

Das Rahmenprogramm „,Forschung für die Nachhaltigkeit" (2005-8) wurde im Juni 2004 durch das Bundeskabinett verabschiedet. FONA soll Beiträge der Forschung zur Umsetzung der Nationalen Nachhaltigkeitsstrategie hervorbringen. Es ist in vier Aktionsfelder gegliedert: (a) Nachhaltigkeit in Industrie und Wirtschaft, (b) Nachhaltige Nutzungskonzepte für Regionen, (c) Nachhaltige Nutzung von natürlichen Ressourcen und

(d) Gesellschaftliches Handeln in Richtung Nachhaltigkeit. Maßnahmen zur Kommunikation und zum Wissenstransfer und eine internationale Strategie ergänzen die Aktionsfelder. Für das Rahmenprogramm sollen jährlich 160 Millionen Euro zur Verfügung stehen.

\section{Weitere Informationen: \\ - Das Rahmenprogramm: www.bmbf.de/pub/forschung_nachhaltigkeit.pdf \\ - BMBF-Internetportal zum Rahmenprogramm www.fona.de \\ - BMBF-Förderschwerpunkt Sozial-ökologische Forschung www.sozial-oekologische-forschung.org}

deutlich, dass Begleitung die Reflexivität der Forschungsorganisation erhöht, eine Weiterentwicklung der Forschungsmethodik unterstïtzen und die Bildung von scientific communities fördern können. Begleitmaßnahmen sind also eine wichtige Komponente der Forschungsförderung für eine Nachhaltige Entwicklung. Ziele, Methoden und institutionelle Verankerung der verschiedenen Begleitaktivitäten zeigen deutliche Unterschiede. Eine vergleichende, systematische Auswertung der Erfahrungen in den verschiedenen Förderschwerpunkten - auch unter Einbeziehung der Forschenden und Praxisakteure - könnte wichtige Impulse für die weitere, zielorientierte Ausgestaltung von Begleitungen in FONA ergeben.

\section{Verlässliche Finanzausstattung erforderlich}

Alle Konzepte sind wohlfeil, wenn Mittel für ihre Umsetzung fehlen. 160 Millionen Euro jährlich will das BMBF nach eigenen Angaben für die Projektförderung in FONA ausgeben. Das klingt viel versprechend, die Summe steht aber ausdrücklich unter Finanzierungsvorbehalt. Im Entwurf für den BMBF-Haushalt 2005 sind Gelder aus der Eigenheimzulage bereits fest eingeplant. Scheitert deren Abschaffung am Bundesrat - aus heutiger Sicht ein wahrscheinliches Szenario - fehlen im Bereich Projektförderung erhebliche Mittel. Für neue Förderinitiativen im Rahmen von FONA wie könnten nächstes Jahr die finanziellen Spielräume fehlen. Die Bundesregierung ist aufgefordert, eine verlässliche Finanzausstattung von FONA zu gewährleisten.

Mit FONA hat das BMBF eine anspruchsvolle Konzeption zur Förderung der transdisziplinären Nachhaltigkeitsforschung in Deutschland vorgelegt. Die Umsetzungspraxis wird zeigen, inwieweit die Bundesregierung den darin vorgelegten Ansprüchen gerecht wird. Das betrifft auch eine gezielte Integration der Ergebnisse und Methoden von FONA in andere Förderbereiche des BMBF - wie beispielsweise die Forschung zu Bio- oder Nanotechnologien. Dies fordert auch der Deutsche Bundestag in seinem Beschluss zu FONA (5), in ähnlicher Weise argumentiert er in seiner Entschließung zur Erforschung der Nanotechnologien (6). In FONA liegt zudem ein Potenzial zur nachhaltigkeitsorientierten Gestaltung der Innovationsoffensive der Bundesregierung, die in konzeptioneller Leere zwischen traditioneller Technikförderung und einem eindimensionalen Begriff von Wettbewerbsfähigkeit verkümmert. Ihr könnte durch die Verknüpfung des Leitbildes einer Nachhaltigen Entwicklung mit einem erweiterten gesellschaftlichen Innovationsbegriff wie er auch FONA zueigen ist neues Leben zuwachsen.

\section{Anmerkungen}

(1) BMBF (Hg.): Forschung für die Nachhaltigkeit. Rahmenprogramm des BMBF für eine zukunftsfähige innovative Gesellschaft. Bonn 2004.

(2) Rat für Nachhaltige Entwicklung (Hg.): Nachhaltigkeit im Visier - Stellungnahme des Rates. Berlin 2004.

(3) ökoforum (Hg.): Critical Analysis of the Sixth Framework Programme for Research and Development from the Perspective of Transdisciplinary Research. Berlin 2001, Download: www.oekoforum.org

(4) www.ina-netzwerk.de und www.sozial-oekologische-forschung.org

(5) Forschung für Nachhaltigkeit - Motor für Innovationen. Bundestagsdrucksache 15/3452 vom 30.06.2004

(6) Aufbruch in den Nanokosmos - Chancen nutzen, Risiken abschätzen. Bundestagsdrucksache 15/3051vom 05.05.2004

\section{Der Autor}

Thomas Korbun ist wissenschaftlicher Geschäftsführer des Instituts für ökologische Wirtschaftsforschung (IÖW).

Kontakt: IÖW, Potsdamer Str. 105, 10785 Berlin. Tel. 030-8845940, E-Mail: thomas.korbun@ioew.de 
(c) 20I0 Authors; licensee IÖW and oekom verlag. This is an article distributed under the terms of the Creative Commons Attribution Non-Commercial No Derivates License (http://creativecommons.org/licenses/by-nc-nd/3.o/), which permits unrestricted use, distribution, and reproduction in any medium, provided the original work is properly cited. 